Research Article

Genomics and Bioinformatics

\title{
Methodological differences can affect sequencing depth with a possible impact on the accuracy of genetic diagnosis
}

\author{
Murilo G. Borges $^{1,2,3}$ (1) , Cristiane S. Rocha ${ }^{1,2}$, Benilton S. Carvalho ${ }^{2,4}$ and Iscia Lopes-Cendes ${ }^{1,2}$ \\ ${ }^{1}$ Universidade Estadual de Campinas (UNICAMP), Faculdade de Ciências Médicas, Departamento de \\ Genética Médica e Medicina Genômica, Campinas, SP, Brazil. \\ ${ }^{2}$ Instituto Brasileiro de Neurociência e Neurotecnologia (BRAINN), Campinas, SP, Brazil. \\ ${ }^{3}$ Universidade Estadual de Campinas (UNICAMP), Instituto de Física "Gleb Wataghin”. Campinas, SP, \\ Brazil. \\ ${ }^{4}$ Universidade Estadual de Campinas (UNICAMP), Instituto de Matemática, Estatística e Computação \\ Cientifica, Departamento de Estatística, Campinas, SP, Brazil.
}

\begin{abstract}
For a better interpretation of variants, evidence-based databases, such as ClinVar, compile data on the presumed relationships between variants and phenotypes. In this study, we aimed to analyze the pattern of sequencing depth in variants from whole-exome sequencing data in the 1000 Genomes project phase 3, focusing on the variants present in the ClinVar database that were predicted to affect protein-coding regions. We demonstrate that the distribution of the sequencing depth varies across different sequencing centers (pair-wise comparison, $p<0.001$ ). Most importantly, we found that the distribution pattern of sequencing depth is specific to each facility, making it possible to correctly assign $96.9 \%$ of the samples to their sequencing center. Thus, indicating the presence of a systematic bias, related to the methods used in the different facilities, which generates significant variations in breadth and depth in whole-exome sequencing data in clinically relevant regions. Our results show that methodological differences, leading to significant heterogeneity in sequencing depth, may potentially influence the accuracy of genetic diagnosis. Furthermore, our findings highlight how it is still challenging to integrate results from different sequencing centers, which may also have an impact on genomic research.
\end{abstract}

Keywords: Whole exome sequencing, depth, ClinVar, computational biology, clinical genomics.

Received: August 28, 2019; Accepted: February 16, 2020.

\section{Introduction}

Whole exome sequencing (WES) has emerged as a powerful tool in genomic medicine as it provides the possibility of interrogating the genome in its most interpretable portion (Coffey et al., 2011; Mahon, 2016). This strategy has identified causal variants in several Mendelian diseases with a high success rate (Gilissen et al., 2012; Shamseldin et al., 2017). Therefore, the use of WES has proven to add relevant diagnostic information, and it is currently widely used in medical practice (Linderman et al., 2014; Suwinski et al., 2019; Ulintz et al., 2019). However, several methodological issues can affect the results obtained by WES and may influence its interpretation (Sulonen et al., 2011; Hardwick et al., 2017).

The capture experiment, followed by the enrichment phase, is a crucial step to ensure success in WES since it is

Send correspondence to Iscia Lopes-Cendes, Universidade Estadual de Campinas (UNICAMP), Faculdade de Ciências Médicas, Departamento de Genética Médica e Medicina Genômica, R. Tessália Vieira de Camargo, 126 - Cidade Universitária Zefererino Vaz, CEP: 13083-887, Campinas, SP, Brazil. E-mail: icendes@unicamp.br essential to determine reads uniformity, depth, and overall quality of sequencing (Chilamakuri et al., 2014; Wang et al., 2017). One of several parameters used for quality control on massively parallel DNA sequencing experiments is the depth of coverage, which refers to the average number of sequenced and adequately aligned bases or reads to a specific genomic position or region (Elmas et al., 2018). Its expected value is one of the first parameters to be estimated in the study design of a given sequencing experiment (Sims et al., 2014). In WES, the depth varies greatly, so that even when the expected average depth is high, the capture of some regions may still be problematic, leading to an uneven distribution of sequencing depth (Lelieveld et al., 2015). It is well-known that the results obtained from massively parallel DNA sequencing technologies may suffer some biases due to the experimental design, sample selection, sequencing strategies, and variant calling methods (Asan et al., 2011; Hwang et al., 2016; Meienberg et al., 2016; Van Allen et al., 2016). In this context, we aimed to analyze the pattern of sequencing depth in variants from WES data in the 1000 Genomes project phase 3, focusing on the variants present in 
the ClinVar database that were predicted to affect proteincoding regions.

\section{Materials and Methods}

We used the public binary alignment map files (BAM) available from the 1000 Genomes Project Consortium FTP web page to calculate the depth of sequencing variations from ClinVar entries in 1,112 WES samples from sequencing phase 3 (Table S1). We guarantee the integrity of the analyzed BAM files by automatically generating and checking the MD5 code of each downloaded file by implementing an automatic script. If there were any discrepancies between the MD5 codes provided by the 1000 Genomes Project and the one obtained by us, we performed the download once again. The samples were all sequenced in an Illumina HiSeq 2000 with a paired-end sequencing reaction in four different sequencing facilities listed below. Each center participating in the consortium applied a different WES capture methodology: the Baylor College of Medicine (BCM) applied a customized array HSGC VCRome, the Broad Institute (BI) used Agilent SureSelect All Exon v2, the Beijing Genomics Institute (BGI) used NimbleGenSeqCap EZ Exome v2, and the Washington University Genome Center (WUGC) used NimbleGenSeqCap EZ Exome v3.

We extracted 282,453 variants from ClinVar (built 20170801, GRCh37.p13) (Landrum et al., 2018) and performed variant annotation using the Ensembl Variant Effect Predictor (VEP version 84) using the default parameters (McLaren et al., 2016). Overall, 4,543 variants were classified as exonic in the autosome chromosomes and had a predicted impact on mRNA and protein structure and function (121 were classified as high, 2,166 moderate, 1,641 low, and 615 as a modifier). We provide the variant calling file containing these targets as File S2. We used "samtools depth" (version 1.3.1) to estimate the base-by-base depth of the 4,543 selected variants for each of the BAM files, accepting reads with sequencing and mapping quality greater than 30 (99.9\% reliability) (Li et al., 2009; $\mathrm{Li}, 2011)$. We then performed the merging of each of the BAM files with the coverage of our ClinVar targets.

We conducted all further analyses using the R statistical environment (version 3.3.2) (R Core Team, 2014). First, we tested the assumption of no difference in the pattern of sequencing depths in each of the four sequencing centers with a Mann-Whitney-Wilcoxon test with continuity correction in the normal approximation for the p-value. We also applied a multidimensional scaling (MDS) method over the resulting depth in each region and compared the different groups, addressing the data high-dimensionality issue, and obtained a low-dimensional representation of the data (Kruskal and Wish, 1978). We show the results obtained using $\mathrm{R}$ packages to process and generate conventional and interactive charts (plyr 1.8.4, plotly 4.8.0, ggplot2 3.0.0). Furthermore, we visually recorded the variation in depth of sequencing in the different sequencing centers with a heatmap (heatmaply 0.9 .1 ) of the 450 variants, which presented the higher variance across samples. We apply a method of clustering to this high variability subset of targets by using the k-means algorithm, considering a total of 5 groups (Macqueen, 1967).

\section{Results}

The average sequencing depth from the selected 4,543 variants from ClinVar differed significantly among the sequencing centers (pairwise comparisons with MannWhitney-Wilcoxon test, $p<0.001$ ), with an average depth of $82.8 \pm 67.6$ for the BCM, $123.0 \pm 85.6$ for the BGI, $86.6 \pm$ 79.2 for the BI, and $49.4 \pm 33.8$ for the WUGSC (Figure 1A, File $\mathrm{S} 2$ ).

The multidimensional scaling analysis corroborates that the pattern of sequencing depth clusters according to each sequencing center, with $69 \%$ of the variance explained by the first two principal components in the principal component analysis (PCA, Figure 1B, File S2). This indicates that protocol advancement and intrinsic methodological dif-

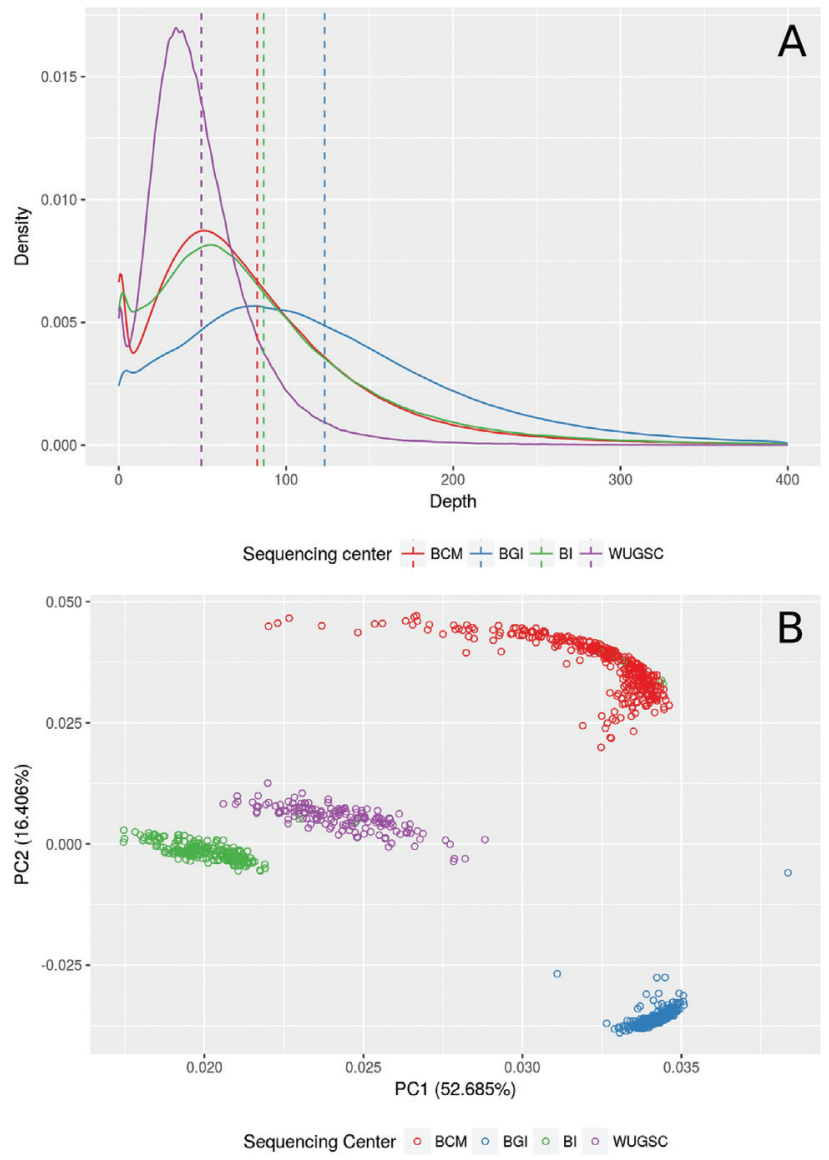

Figure 1 - Variation in depth of sequencing in different sequencing centers. Depth distribution varies significantly $(p<0.001)$ among samples from the four sequencing centers included in this study (BCM - Baylor College of Medicine, BI - Broad Institute, BGI, and WUGC - Washington University Genome Center). (A) Density distributions for regions from ClinVar with depth from 0 to 400 , with an average of $82.8 \pm 67.6$ for BCM, $123.0 \pm 85.6$ for BGI, $86.6 \pm 79.2$ for BI, and $49.4 \pm 33.8$ for WUGSC. (B) Principal component analysis (PCA) corroborates our findings, with an explained variance of $69.0 \%$ for the first two components. Complete depth distribution and an interactive $3 \mathrm{D}$ version of Figure 1B is available as File S3. 
ferences in each of the sequencing centers directly affect the pattern of the sequencing depth in the set of variants analyzed. The inconsistency in the depth and breadth of coverage across samples introduces a systematic bias in the results generated by each center. Sequencing depth is a measurement of how many times a certain variant was sequenced while the breadth is the capability of adequately capturing and sequencing a given region.

Furthermore, by analyzing the distribution of the sequencing depth of the 450 variants with higher variance, we could correctly assign $96.9 \%$ of the samples to their sequencing center when considering five clusters to the dendrogram branches depicted in Figure 2 and File S3. This finding also supports the existence of different coverage patterns for each sequencing center, evidenced in the individual coverage of each of the samples considered in these analyses.

\section{Discussion}

Understanding how the depth of sequencing varies in sequencing experiments is essential to find a balance between the number of reads necessary to answer a genetic question and the costs and efforts required to do so (Sims et al., 2014; Meienberg et al., 2015; Lek et al., 2016). The use of WES over WGS reduces the broad genomic region to be analyzed, dropping costs and allowing it to be more widely used in medical practice (Hu et al., 2017; Manrai et al.,
2018; Suwinski et al., 2019). The public availability of data from large genomic projects performed by worldwide consortia, such as ExAC, ESP, 1000 Genomes Project, UK10K, and GoNL, is of the utmost importance for both research and medical applications of these technologies (van Rooij et al., 2017). However, one should consider the existence of methodological covariates that may introduce potential bias into the sequencing data. In our case, the possible false-negatives, which could, for example, mask the allelic frequency of a given variant returned from a sequencing center. Thus, we note the possibility of considering certain variants as "false-rares," since their frequency would be diminished in the variant discovery process (Schaid et al., 2018).

Kong et al. (2018) argue that both researchers and patients could benefit from clearer methodological specifications from vendors. We agree and believe that initiatives that propose the public availability of data should also provide as many technical informaion as possible. This could help users to evaluate better any bias related to the technique or methodology used to generate or to interpret the data, which could lead to erroneous or discordant clinical interpretations, for example. Here, we focused on variants that are likely to have clinical significance (comprising of 4,543 variants), since they were predicted to promote mRNA changes and/or protein structure and function alterations related to a phenotype described in ClinVar (File S2). By doing so, we aimed to as-

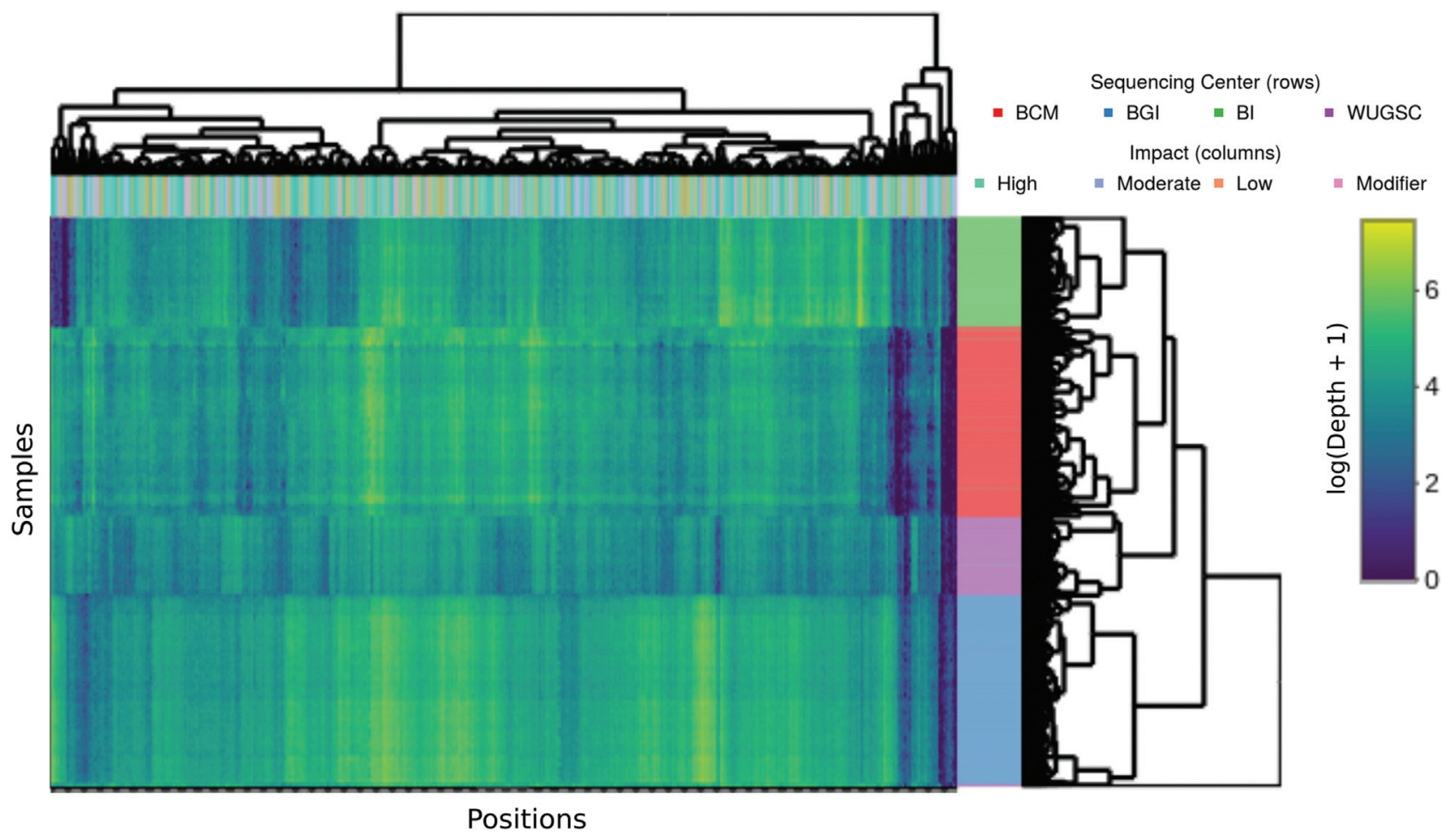

Figure 2 - Variation in sequencing depth across sequencing centers and coding impact. Heatmap showing variation in depth across sequencing centers for the 450 variants with higher variance across samples. Each row represents a sample from one of the sequencing centers (BCM - Baylor College of Medicine, BI - Broad Institute, BGI, and WUGC - Washington University Genome Center). 96.9\% of samples are correctly assigned to their sequencing centers when considering five clusters to the dendrogram branches. The columns represent each one of the variants, with their impact classified as high, moderate, low, or modifier, which is an indicator that the coding impact does not influence the depth of coverage ( $\mathrm{p}>0.05$ for each pair comparisons). An interactive version of this figure is available as File S4. 
sess the potential impact of variability in sequencing depth on genetic diagnosis performed by WES. This is especially relevant when a diagnostic test fails to report a variant since this could indicate either a true negative, when the genomic position of the variant is adequately captured and sequenced or a false negative when the variant is not captured or appropriately sequenced (Patwardhan et al., 2015; Shigemizu et al., 2015; Karlsson Linnér et al., 2019).

Our results indicate that the distribution of sequencing depth varied across different sequencing centers from the 1000 Genomes Project, phase 3 (pairwise comparisons, $\mathrm{p}<$ 0.001). Most importantly, we found that there is a pattern of distribution in sequencing depth, which is specific to each facility (Figure 1). These findings are evidenced by the clustering of samples by PCA (69\% of variance explained) and clustering of more than $95 \%$ of the samples to their sequencing centers when considering sites with highly variant coverage. These findings indicate that these patterns may be related to the methodologies used by each center. It is certainly likely that there are specific regions that differentially failed to generate adequate coverage, either due to design or capture efficiency (Altmüller et al., 2016; García-García et al., 2016). That means that a variant could be missed in any specific patient who was sequenced using a certain methodology specific to the sequencing center where the experiment was conducted, generating a serious problem imposed on clinical sequencing. One other piece of evidence that corroborates this is the wide standard deviation found for each of the sequencing facilities, indicating an unspecific capture reaction. The inconsistency in the breadth and depth across the targets comprising of the medically relevant variants demands the attention of professionals and patients seeking diagnosis by WES. Such an example happens with the establishment of the expanded or clinical exome capturing kits, which tend to maximize variant discovery resolution, but potentializes capture bias as well (Shamseldin et al., 2017; Suwinski et al., 2019). This finding also raises questions about the low frequency of a given variant that may be due to the methodological bias described in this work.

When performing WES, a critical experimental step is the capture reaction. It is well known that the efficiency of capture depends on several experimental procedures as well as on probe design, which may directly affect sequence depth and uniformity (Do et al., 2012; Chandler et al., 2016). Therefore, problems in the capturing reaction directly affect the final experiment results, yielding not only regions with different average depths but also leading to regions with no coverage at all (Lionel et al., 2018; Wang et al., 2018). We demonstrated here that differences, most likely attributed to the different methods used by the sequencing centers, proved to play a significant role in determining the distribution of sequencing depth in WES data from the 1000 Genomes Project. We understand that the methodological variability in the 1000 Genome Consortium could be the best way to achieve a more in-depth and broader variant catalog capable of establishing the bases to understand population allele frequency; however, it is also important to recognize the limita- tions imposed by the methods used. This finding represents a challenge for large or long-term exome sequencing projects that expect to aggregate advancements in capture techniques over time (McCarthy and MacArthur, 2017; Sanghvi et al., 2018). In addition, it poses questions about the reproducibility of results among different diagnostic laboratories performing WES, indicating the need for further discussion about the use of clear open methods (both from the wet and dry lab), which could minimize such bias (Eberle et al., 2017; Haga, 2017; Roy et al., 2018). The proposal of returning information not only on the variants identified but also about the methods used, including the regions analyzed and all the characteristics of the sequencing reaction, could minimize misinterpretation, which directly influences the accuracy of genetic testing.

\section{Conclusions}

Our results indicate that the sequencing depth in WES varies significantly across different facilities, leading to a systematic bias, which is most likely introduced by technical differences. Our findings indicate that the low coverage or lack of consistency between WES methodologies has direct clinical applications. It may introduce false-negatives into experiments performed for diagnostic purposes and results in variants with a lower frequency than expected. Our results are not surprising, given that the initial step for a WES experiment is the capture of the target regions to be subsequently enriched and sequenced and that this step is susceptible to the effects of many technical factors. Although difficult to address, the issue of standardized and open methodologies should be further discussed.

\section{Acknowledgments}

The authors thank Prof. Vera Solferini, Prof. Plinio Barbosa, Ph.D. Ticiana Mira, and Dr. Joana Prota for their contributions on technical assistance and critical review of the manuscript. This work was supported by Fundação de Amparo à Pesquisa do Estado de São Paulo (FAPESP), grant \#2013/07559-3; High-Performance National Center (CENAPAD-Campinas), Sao Paulo, Brazil), project "proj595" (UNICAMP / FINEP - MCT) and Coordenação de Aperfeiçoamento de Pessoal de Nível Superior - Brasil (CAPES), grant \# 001.Dr. IL-C is supported by Conselho Nacional de Pesquisa (CNPq), Brazil, grants \# 309494/2014-1 and 403299/2016-0.

\section{Conflict of Interests}

The authors declare that they have no competing interests.

\section{Author Contributions}

MGB conceived the study, designed and performed the statistical analysis, and wrote the manuscript. CSR and BSC participated in the design of the study and helped to develop the statistical code. IL-C participated in the design and 
coordination of the study and helped to draft the manuscript. All authors have read and approved the final manuscript.

\section{References}

Altmüller J, Motameny S, Becker C, Thiele H, Chatterjee S, Wollnik B and Nürnberg P (2016) A systematic comparison of two new releases of exome sequencing products: The aim of use determines the choice of product. Biol Chem 397:791801.

Asan, Xu Y, Jiang H, Tyler-Smith C, Xue Y, Jiang T, Wang J, Wu M, Liu X, Tian G et al. (2011) Comprehensive comparison of three commercial human whole-exome capture platforms. Genome Biol 12:R95.

Chandler MR, Bilgili EP and Merner ND (2016) A review of whole-exome sequencing efforts toward hereditary breast cancer susceptibility gene discovery. Hum Mutat 37:835-846.

Chilamakuri CSR, Lorenz S, Madoui MA, Vodák D, Sun J, Hovig E, Myklebost O and Meza-Zepeda LA (2014) Performance comparison of four exome capture systems for deep sequencing. BMC Genomics 15:449.

Coffey AJ, Kokocinski F, Calafato MS, Scott CE, Palta P, Drury E, Joyce CJ, LeProust EM, Harrow J, Hunt S et al. (2011) The GENCODE exome: Sequencing the complete human exome. Eur J Hum Genet 19:827-831.

Do R, Kathiresan S and Abecasis GR (2012) Exome sequencing and complex disease: Practical aspects of rare variant association studies. Hum Mol Gen 15:R1-R9.

Eberle MA, Fritzilas E, Krusche P, Källberg M, Moore BL, Bekritsky MA, Iqbal Z, Chuang HY, Humphray SJ, Halpern AL et al. (2017) A reference data set of 5.4 million phased human variants validated by genetic inheritance from sequencing a three-generation 17-member pedigree. Gen Res 27:157-164.

Elmas M, Yyldyz H, Erdodan M, Gogus B, Avcy K and Solak M (2018) Comparison of clinical parameters with whole exome sequencing analysis results of autosomal recessive patients: a center experience. Mol Biol Rep 46:287-299.

García-García G, Baux D, Faugère V, Moclyn M, Koenig M, Claustres M and Roux AF (2016) Assessment of the latest ngs enrichment capture methods in clinical context. Sci Rep 6:20948.

Gilissen C, Hoischen A, Brunner HG and Veltman JA (2012) Disease gene identification strategies for exome sequencing. Eur J Hum Genet 20:490-497.

Haga SB (2017) Update: Looking beyond the 100,000 genome project. Pers Med 14:85-87.

Hardwick SA, Deveson IW and Mercer TR (2017) Reference standards for next-generation sequencing. Nat Rev Genet 18:473-484.

Hu Y, Lu Q, Powles R, Yao X, Yang C, Fang F, Xu X and Zhao H (2017) Leveraging functional annotations in genetic risk prediction for human complex diseases. PLOS Comp Biol 13:e1005589.

Hwang S, Kim E, Lee I and Marcotte EM (2016) Systematic comparison of variant calling pipelines using gold standard personal exome variants. Sci Rep 5:17875.

Karlsson Linnér R, Biroli P, Kong E, Meddens SFW, Wedow R, Fontana MA, Lebreton M, Tino SP, Abdellaoui A, Hammerschlag AR et al. (2019) Genome-wide association analyses of risk tolerance and risky behaviors in over 1 million individuals identify hundreds of loci and shared genetic influences. Nat Genet 51:245-257.
Kong SW, Lee IH, Liu X, Hirschhorn JN and Mandl KD (2018) Measuring coverage and accuracy of whole-exome sequencing in clinical context. Genet Med 20:1617-1626.

Kruskal JB and Wish M (1978) Quantitative Applications in the Social Sciences: Multidimensional scaling. SAGE Publications, Thousand Oaks.

Landrum MJ, Lee JM, Benson M, Brown GR, Chao C, Chitipiralla S, Gu B, Hart J, Hoffman D, Jang W et al. (2018) ClinVar: Improving access to variant interpretations and supporting evidence. Nucleic Acids Res 46:D1062-D1067.

Lek M, Karczewski KJ, Minikel EV, Samocha KE, Banks E, Fennell T, O'Donnell-Luria AH, Ware JS, Hill AJ, Cummings $\mathrm{BB}$ et al. (2016) Analysis of protein-coding genetic variation in 60,706 humans. Nature 536:285-291.

Lelieveld SH, Spielmann M, Mundlos S, Veltman JA and Gilissen C (2015) Comparison of exome and genome sequencing technologies for the complete capture of protein-coding regions. Hum Mut 36:815-822.

Li H (2011) A statistical framework for SNP calling, mutation discovery, association mapping and population genetical parameter estimation from sequencing data. Bioinformatics 27:2987-2993.

Li H, Handsaker B, Wysoker A, Fennell T, Ruan J, Homer N, Marth G, Abecasis G and Durbin R (2009) The sequence alignment/map format and samtools. Bioinformatics 25:20782079.

Linderman MD, Brandt T, Edelmann L, Jabado O, Kasai Y, Kornreich R, Mahajan M, Shah H, Kasarskis A and Schadt EE (2014) Analytical validation of whole exome and whole genome sequencing for clinical applications. BMC Med Geno$\operatorname{mics} 7: 20$.

Lionel AC, Costain G, Monfared N, Walker S, Reuter MS, Hosseini SM, Thiruvahindrapuram B, Merico D, Jobling R, Nalpathamkalam $\mathrm{T}$ et al. (2018) Improved diagnostic yield compared with targeted gene sequencing panels suggests a role for whole-genome sequencing as a first-tier genetic test. Genet Med 20:435-443.

Macqueen J (1967) Some methods for classification and analysis of multivariate observations. In: Procedings of the Fifth Berkeley Symposium on Mathematical Statistics and Probability, Berkley, p. 281-297

Mahon S (2016) Whole exome sequencing: The next phase of genetics care. Oncol Nurs Forum 43:249-252.

Manrai AK, Patel CJ and Ioannidis JPA (2018) In the era of precision medicine and big data, who is normal? JAMA 319:1981-1982.

McCarthy MI and MacArthur DG (2017) Human disease genomics: From variants to biology. Gen Biol 18:20.

McLaren W, Gil L, Hunt SE, Riat HS, Ritchie GRS, Thormann A, Flicek P and Cunningham F (2016) The ensembl variant effect predictor. Genome Biol 17:122.

Meienberg J, Bruggmann R, Oexle K and Matyas G (2016) Clinical sequencing: Is WGS the better WES? Hum Genet 135:359.

Meienberg J, Zerjavic K, Keller I, Okoniewski M, Patrignani A, Ludin K, Xu Z, Steinmann B, Carrel T, Röthlisberger B et al. (2015) New insights into the performance of human whole-exome capture platforms. Nucleic Acids Res 43:e76.

Patwardhan A, Harris J, Leng N, Bartha G, Church DM, Luo S, Haudenschild C, Pratt M, Zook J, Salit M et al. (2015) Achieving high-sensitivity for clinical applications using augmented exome sequencing. Gen Med 7:71.

Roy S, Coldren C, Karunamurthy A, Kip NS, Klee EW, Lincoln SE, Leon A, Pullambhatla M, Temple-Smolkin RL, Voelkerding KV et al. (2018) Standards and guidelines for 
validating next-generation sequencing bioinformatics pipelines: A joint recommendation of the association for molecular pathology and the college of American pathologists. J Mol Diagn 20:4-27.

Sanghvi RV, Buhay CJ, Powell BC, Tsai EA, Dorschner MO, Hong CS, Lebo MS, Sasson A, Hanna DS, McGee S et al. (2018) Characterizing reduced coverage regions through comparison of exome and genome sequencing data across 10 centers. Genet Med 20:855-866.

Schaid DJ, Chen W and Larson NB (2018) From genome-wide associations to candidate causal variants by statistical fine-mapping. Nat Rev Genet 19:491-504.

Shamseldin HE, Maddirevula S, Faqeih E, Ibrahim N, Hashem M, Shaheen R and Alkuraya FS (2017) Increasing the sensitivity of clinical exome sequencing through improved filtration strategy. Genet Med 19:593-598.

Shigemizu D, Momozawa Y, Abe T, Morizono T, Boroevich KA, Takata S, Ashikawa K, Kubo M and Tsunoda T (2015) Performance comparison of four commercial human whole-exome capture platforms. Sci Rep 5:12742.

Sims D, Sudbery I, Ilott NE, Heger A and Ponting CP (2014) Sequencing depth and coverage: Key considerations in genomic analyses. Nat Rev Genet 15:121-132.

Sulonen AM, Ellonen P, Almusa H, Lepistö M, Eldfors S, Hannula $\mathrm{S}$, Miettinen T, Tyynismaa H, Salo P, Heckman C et al. (2011) Comparison of solution-based exome capture methods for next generation sequencing. Gen Biol 12:R94.

Suwinski P, Ong C, Ling MHT, Poh YM, Khan AM and Ong HS (2019) Advancing personalized medicine through the application of whole exome sequencing and big data analytics. Front Genet 10:49.

Ulintz PJ, Wu W and Gates CM (2019) Bioinformatics Analysis of Whole Exome Sequencing Data. Methods Mol Biol 1881:277-318.

Van Allen EM, Robinson D, Morrissey C, Pritchard C, Imamovic A, Carter S, Rosenberg M, McKenna A, Wu YM, Cao X et al. (2016) A comparative assessment of clinical whole exome and transcriptome profiling across sequencing centers: Implications for precision cancer medicine. Oncotarget 7:52888-52899.

van Rooij JGJ, Jhamai M, Arp PP, Nouwens SCA, Verkerk M, Hofman A, Ikram MA, Verkerk AJ, van Meurs JBJ, Rivadeneira F et al. (2017) Population-specific genetic variation in large sequencing data sets: Why more data is still better. Eur J Hum Genet 25:1173-1175.

Wang Q, Shashikant CS, Jensen M, Altman NS and Girirajan S (2017) Novel metrics to measure coverage in whole-exome sequencing datasets reveal local and global non-uniformity. Sci Rep 7:885.
Wang VG, Kim H and Chuang JH (2018) Whole-exome sequencing capture kit biases yield false-negative mutation calls in TCGA cohorts. PLoS One 13:e0204912.

\section{Internet Resources Section}

The International Genome Sample Resource: "What capture technology did the Exome sequencing use?", http://www.internationalgenome.org/faq/what-capture-techn ology-does-exome-sequencing-used/ (accessed 10 August 10 2019).

R Core Team (2014) R: A language and environment for statistical computing, https://www.R-project.org (accessed 10 August 2019).

\section{Supplementary material}

The following online material is available for this article:

Table S1. Detailed information on the public data we used from the 1000 Genomes Project Consortium (doc).

File S2. Variant calling file containing 4,543 variants from ClinVar (vcf). We extracted 282,453 variants from ClinVar (built 20170801, GRCh37.p13) and performed variant annotation using the Ensembl Variant Effect Predictor (VEP version 84) using the default parameters. Four thousand five hundred forty-three variants were classified as exonic and had a predicted impact on function (121 were classified as high, 2,166 moderate, 1,641 low, and 615 as a modifier).

File S3. Distribution of depth and PCA analysis for different sequencing centers, as depicted in Figure 1 (HTML). Figure $1 \mathrm{~A}$ shows a complete distribution of depth of sequencing and an interactive 3D version of Figure 1B. Better visualized in Google Chrome.

File S4. Variation in depth across sequencing centers and coding impact data from Figure 2 (HTML). Heatmap showing the variation of depth across sequencing centers of the 450 variants with higher variance. Each row represents a sample from one of the sequencing centers (BCM - Baylor College of Medicine, BI - Broad Institute, BGI, and WUGC Washington University Genome Center). The columns represent each one of the variants, with their impact classified as high, moderate, low, or modifier. Better visualized in Google Chrome.

Associate Editor: Ana Tereza Vasconcelos

License information: This is an open-access article distributed under the terms of the Creative Commons Attribution License (type CC-BY), which permits unrestricted use, distribution and reproduction in any medium, provided the original article is properly cited. 